\title{
Errata: Effective properties of membrane photonic crystals
}

\author{
Didier Felbacq, ${ }^{\mathrm{a}}$ Brahim Guizal, ${ }^{\mathrm{a}}$ Guy Bouchitté, ${ }^{\mathrm{b}}$ and Christophe \\ Bourel $^{\mathbf{b}}$ \\ ${ }^{a}$ University of Montpellier II, GES UMR 5650, Bat. 21 CC074 Place E. Bataillon, 34095 \\ Montpellier Cedex 05, France \\ dfelbacq@univ-montp2.fr \\ ${ }^{b}$ University of Toulon, ANAM, BP 132, 83957 La Garde Cedex, France
}

This article [J. Nanophoton. 3, 031507 (2009)] was originally published online on 20 February 2009. The following corrections were made to the article on 20 February 2009:

1. Christophe Bourel, University of Toulon, ANAM, BP 132, 83957 La Garde Cedex, France was added as an author.

2. In the first sentence of the Abstract, the word "were" was changed to "was." 